\title{
On the correlation between the X-ray and gamma-ray emission in TeV blazars
}

\author{
K. Katarzyński and K. Walczewska \\ Toruń Centre for Astronomy, Nicolaus Copernicus University, ul. Gagarina 11, 87100 Toruń, Poland \\ e-mail: kat@astro.uni.torun.pl
}

Received 17 August 2009 / Accepted 17 November 2009

\begin{abstract}
Aims. The observations of TeV blazars published recently show an unexpected quadratic or even cubic correlation between the X-ray and gamma-ray emission. A standard model of the synchrotron self-Compton emission of a compact source inside a jet is not able to explain such a correlation. Therefore, we propose an alternative scenario where the emission of at least two independent compact components is observed at the same time.

Methods. We compare two different models. The first model assumes the injection of relativistic particles into a downstream region of a shock wave inside a jet that creates the emitting source. The model precisely describes the evolution of the particle energy spectrum inside the source and takes into account a light-crossing time effect for the produced radiation. The second model assumes an intrinsically constant emission of a homogeneous source that travels inside the jet along a curved trajectory, where the activity is produced simply by different values of the source's Doppler factor. To verify the two models we use recently published observations of Mrk 421.

Results. Our simulations show that simultaneous radiation of at least two independent sources, where the first source dominates the emission in the X-ray range and the second source radiates strongly in the gamma-ray range, can explain the observed correlations. However, the injection model provides inadequate results because it gives different values for the correlation of the rise and decay of a flare. This problem is negligible in the scenario that uses the Doppler boosting effect. Therefore, this approach yields much better results.
\end{abstract}

Key words. radiation mechanisms: non-thermal - galaxies: active - BL Lacertae objects: individual: Mrk 421

\section{Introduction}

Activity of $\mathrm{TeV}$ blazars is usually observed simultaneously in the X-ray and the gamma-ray range (e.g. Catanese et al. 1997; Pian et al. 1998; Sambruna et al. 2000; Takahashi et al. 2000; Krawczynski et al. 2002; Donnarumma et al. 2009). Therefore, the activity can be analysed by a simple comparison of the two different light curves. Such a comparison has shown in a few cases a surprisingly precise correlation between the evolution of the X-ray and the gamma-ray emission. This provides an excellent opportunity to test theoretical models for very high energy emission of blazars.

To describe the correlation is convenient to assume that the evolution of the X-ray or gamma-ray flux during an outburst can be approximated by a power-law function $\left(F_{\mathrm{X}} \propto t^{s}\right.$ and $F_{\mathrm{TeV}} \propto t^{c}$ ) with different values of $s, c$ for the rise and decay phase of the activity. Rewriting the first relationship as $t \propto F_{\mathrm{X}}^{1 / s}$ and substituting this to the second proportionality we can define the correlation

$F_{\mathrm{TeV}} \propto F_{\mathrm{X}}^{c / s}$,

which is also a power-law function with the index $x=c / s$. Such a simple definition can describe precisely the correlations observed in TeV blazars.

The analysis of the light curves produced by different blazars shows that there is no unique value of the correlation slope. The activity of Mrk 501 observed in April 1997 (Catanese et al. 1997) gives $x=1.71 \pm 0.50$ for the comparison between the
50-150 keV light curve obtained by the OSSE experiment and the gamma-ray observations obtained above $350 \mathrm{GeV}$ by the Whipple telescope. On the other hand the comparison between the $2-10 \mathrm{keV}$ observations made by the RXTE-ASM experiment and the gamma rays detected by the Whipple for the same activity of Mrk 501 gives $x=2.69 \pm 0.56$ (Katarzynski et al. 2005). Moreover, observations of Mrk 501 conducted by the RXTE-PCU experiment (2-20 keV) and the gamma-ray telescopes (HEGRA and Whipple) show a linear correlation $(x=$ $0.99 \pm 0.01)$ for the data obtained in May 1997 and an almost quadratic relation $(x=2.07 \pm 0.27)$ for the observations made in June 1998 (Gliozzi et al. 2006).

The observations of Mrk 421 made in March 2001 (Fossati et al. 2008) show several outbursts observed simultaneously in the X-rays (RXTE) and the gamma rays (HEGRA, Whipple). The detailed data analysis performed by the authors of the observations shows at least in one case more than a quadratic correlation $(x>2)$ and in two cases significantly more than linear relation $(x>1)$.

Recent observations of PKS 2155-304 made by Chandra and the H.E.S.S. experiments show a much more steeper correlation with the index $x \simeq 3$ (Acharonian et al. 2009). However, in this particular case the correlation was observed mostly in a decay phase of the flare because of a delay of the Chandra observations. Steep correlation means a relatively small change of the $\mathrm{X}$-ray flux in comparison with the variation of the gamma-ray emission. This means that in an extreme case, where $x \rightarrow \infty$ only 
gamma-ray activity can be observed. This was reported at least two times (e.g. Krawczynski et al. 2004; Blazejowski et al. 2005) and is know in the literature as an orphan flare phenomenon.

The correlation can be well determined only when an activity event is well observed by two different instruments. This requires very good sampling of the recorded light curves (at least several observations during a flare) and small error values in comparison with the amplitude of the variations. These conditions made it possible for only a few cases to precisely determine the index of the correlation so far. So was the correlation sufficiently determined only for three out of nine cases analysed by Fossati et al. (2008). Also more recent observations of $\mathrm{TeV}$ blazars do not give a definitive answer about the correlation slope (e.g. Albert et al. 2007a; Horan et al. 2009; Bonnoli et al. 2009).

The observations show that the correlation slope is changing form linear to cubic. However, the correlations obtained for a relatively long period of the observations (weeks or moths) are usually linear or slightly more than linear (e.g. Gliozzi et al. 2006; Albert et al. 2007b), whereas the observations of short flaring events (a few hours) give quadratic or even cubic relations (e.g. Fossati et al. 2008; Aharonian et al. 2009). Moreover, a scatter of the correlated data points seems to be much higher for the long period correlations in comparison with the results obtained for the short events. The long time correlation contains observations of many different flares produced by different components of a jet, and this is probably the reason of the scatter. Therefore, the short time correlations should provide much better constraints for the emission models. Especially interesting are cases where the short time correlation is quadratic or more than quadratic. Standard one-zone models frequently used to explain the high energy emission of blazars are not able to explain such a slope of the correlation (Katarzynski et al. 2005). In the present work we propose a more complex approach, where the emission of at least two independent sources is observed at the same time.

\section{The problem of the quadratic correlation}

The most simple model that is able to explain the high energy emission of $\mathrm{TeV}$ blazars assumes a compact source located inside the jet at a distance of less that $1 \mathrm{pc}$ from the centre. The source is filed uniformly by relativistic electrons and a tangled magnetic field. The particles spinning around the magnetic field lines are producing a synchrotron emission which is usually observed in the X-ray range. Some fraction of this emission is up-scattered to higher energies by the same population of the electrons. This is the well-known synchrotron self-Compton (hereafter SSC) radiation that appears in the gamma-ray range. This simple scenario was used many times to explain the high energy spectra of TeV blazars (e.g. Bloom \& Marscher 1996; Ghisellini et al. 1996; Inoue \& Takahara 1996; Mastichiadis \& Kirk 1997; Krawczynski et al. 2000; Katarzynski et al. 2001).

The intensity of the synchrotron emission is proportional to the particle density, whereas the intensity of the SSC radiation is proportional to the square of the particle density. This well-known relationship could explain in principle the observed quadratic correlations if only the change of the particle density were responsible for the observed activity. The question is how realistic such a scenario is.

An injection of the relativistic particles into the source, which increases the density, could in principle explain the quadratic correlation during the rising phase of a flare. But the particles should be injected simultaneously into the entire volume of the source. Moreover, the source volume and the magnetic field inside the source should remain constant during the injection phase. The radiative cooling of the particles should also be negligible during the injection. All these requirements render this scenario unrealistic. We will demonstrate that a more realistic scenario, which assumes a local injection into an expanding source where radiative cooling is important, leads to linear correlation during the rising phase.

By analogy systematic energy-independent escape of the particles which decrease the density could in principle explain the quadratic correlation during the decay phase of a flare. This process requires a significantly weaker magnetic field outside the source in order to reduce the efficiency of the synchrotron emission. On the other hand the radiation field energy density outside a spherical ( $R$-radius) homogeneous source at a distance of $1 / 2 R$ above the source surface is only half as weak as on the surface (Gould 1979). This means that particles outside the source can still efficiency produce gamma rays through the inverseCompton scattering. In other words, the gamma-ray emission will not decay fast enough to produce the quadratic correlation during the decay phase.

The detailed analysis of the correlation for many different scenarios of a single source evolution was performed by Katarzynski et al. (2005), where simple analytic formulae were derived to describe basic cases, and more complex scenarios were analysed through numerical simulations. This analysis shows that in all realistic cases we should expect rather linear than quadratic correlation.

Finally, in the case of PK2155-304 the correlation with the index $x \simeq 3$ was observed (Aharonian et al. 2009) and this certainly cannot be explained by the changes of the particle density alone.

\section{Single source vs two sources}

A steep slope of the correlation $(x \gtrsim 2)$ can be easily obtained if we consider simultaneous emission of at least two sources. But let us first assume the emission of a single source, where the X-ray flux is increasing during a rising phase of a flare as a power-law function, where

$F_{\mathrm{X}, \min }=a t_{\min }^{s}, \quad F_{\mathrm{X}, \max }=a t_{\max }^{s}$

is the flux at the beginning $\left(t_{\min }\right)$ and the maximum $\left(t_{\max }\right)$ of the activity respectively. Comparing the two fluxes we can define the ratio of the $\mathrm{X}$-ray fluxes in the rising phase

$F_{\mathrm{X}, \mathrm{r}}=\frac{F_{\mathrm{X}, \text { max }}}{F_{\mathrm{X}, \text { min }}}=\frac{t_{\max }^{s}}{t_{\text {min }}^{s}}$,

and derive the power-law index

$s=\frac{\ln \left(F_{\mathrm{X}, \mathrm{r}}\right)}{\ln \left(t_{\max }\right)-\ln \left(t_{\min }\right)}$.

We can repeat this assumption and calculation for the gammaray emission, which gives the index for the flux evolution in a similar form

$c=\frac{\ln \left(F_{\mathrm{TeV}, \mathrm{r}}\right)}{\ln \left(t_{\max }\right)-\ln \left(t_{\min }\right)}$.

Therefore, the slope of the correlation can be described by a simple formula

$x=\frac{c}{s}=\frac{\ln \left(F_{\mathrm{TeV}, \mathrm{r}}\right)}{\ln \left(F_{\mathrm{X}, \mathrm{r}}\right)}$

that is valid for a single-source emission. 
The formula derived above shows that the correlation slope can be increased by an increase of the gamma-ray flux ratio $\left(F_{\mathrm{TeV}, \mathrm{r}}\right)$ or by a decrease of the X-ray flux radio $\left(F_{\mathrm{X}, \mathrm{r}}\right)$. The first option is limited by the nature of the SSC emission which gives at maximum a quadratic relation in the specific conditions that are rather not realistic, as we discussed already. We decided accordingly to test the second possibility and to decrease the X-ray flux ratio. This ratio will decrease significantly when we assume an additional source of the X-ray emission. In the simplest case we can assume a constant level of this additional emission in a time $F_{\mathrm{X}}$, const. This gives

$F_{\mathrm{X}, \mathrm{r}}^{\prime}=\frac{F_{\mathrm{X}, \max }+F_{\mathrm{X}, \text { const }}}{F_{\mathrm{X}, \min }+F_{\mathrm{X}, \text { const }}} \leqslant \frac{F_{\mathrm{X}, \max }}{F_{\mathrm{X}, \min }}=F_{\mathrm{X}, \mathrm{r}}$

for $F_{\mathrm{X} \text {, const }} \geqslant 0$ and shows that $F_{\mathrm{Xq}}$, const must be comparable or greater than $F_{\mathrm{X} \text {,min }}$ to significantly modify the ratio. On the other hand, possible gamma-ray emission of this additional source should be negligibly small in comparison with the first source emission to avoid simultaneous modification of $F_{\mathrm{TeV}, \mathrm{r}}$. Moreover, with additional X-ray emission the correlation is no longer a simple power-law function and Eq. (6) can be used only for the estimation of the correlation slope. But the derived formulae explain why the correlation slope can be steeper also in more complex situations which are difficult to describe using simple analytic relations.

\section{The observations}

In this work we focus on the activity of Mrk 421 observed during the campaign of observations conducted in March 2001 by RXTE, HEGRA and the Whipple experiments (Fossati et al. 2008). The seven days long light curves obtained during this campaign were divided into nine periods, and the activity events were analysed separately. The best results were obtained for the first, fourth and fifth night of the observations (March 18/19, 21/22, 22/23).

More than quadratic correlation was observed only during the first night. The comparison of the X-ray flux $(0.2-10 \mathrm{keV})$ and all the available $\mathrm{TeV}$ observations $(E>0.4 \mathrm{TeV})$ give in this case $x=2.26 \pm 0.25$. But the comparison between the $\mathrm{X}$-ray data and the $\mathrm{TeV}$ observations made only by Whipple during this night gives the almost cubic value of the correlation $x=2.84 \pm 0.41$. What is important, only Whipple was observing a strong flare during this night, the light curve obtained by HEGRA before the flare shows no activity at all.

The correlation obtained for the fourth and fifth night was less than quadratic but significantly more than linear with $x=$ $1.56 \pm 0.25$ and $x=1.67 \pm 0.16$ respectively. Note that the relative amplitudes of the flux changes are significantly lower in these observations.

The flare observed during the first night seems to be one of the best activity events ever observed in TeV blazars. The evolution of the X-ray and gamma-ray flux was well detected during the rise and the decay phase of the flare. This gives very important information about the correlation which seems to be very similar for the rising and decay phase. Moreover, the excellent observations give also information about the spectral evolution of the emission. Finally, this activity seems to be quite simple, created by one or two sources, whereas the light curves obtained during the other nights looks like a superposition of many events in time. We consequently selected this particular flare to test our models. If a model is able to explain the correlation obtained for this extreme flare, it should be able to explain any other correlation as well.

\section{Injection scenario}

To explain the correlation it is necessary to apply time-dependent modeling. Many different models have been proposed to explain the evolution of the high energy activity in TeV blazars so far (e.g. Dermer 1997; Kirk et al. 1998; Coppi \& Aharonian 1999; Kataoka 2000). Most of them assume changes in the particle energy spectrum inside a source as the main reason of the activity. Some more complex models additionally are taking into account the light-crossing time effect (e.g. Chiaberge \& Ghisellini 1999; Sokolov et al. 2004; Graff et al. 2008).

In the first part of this work we use the relatively simple model proposed by Chiaberge \& Ghisellini (1999) and improved by Katarzynski et al. (2008). The model assumes particle acceleration by a shock wave inside a jet. The shock is created by two or more colliding components of the jet, where some fraction of the kinetic energy of the components is converted into random energy of the particles. This is the well-known internal shock scenario proposed for the first time by Rees (1978). However, there is no precise description of the acceleration process in the model we use. The particle energy increases relatively fast at the shock region and after that most of the particles escape into the downstream region of the shock. Therefore, the acceleration can be approximated as an injection of the particles into some volume. In this particular case we assume a power-law energy distribution of the injected particles $Q(\gamma)=Q_{\text {inj }} \gamma^{-n}$, where the particle energy is given by $E=\gamma m_{\mathrm{e}} c^{2}$. The geometry of the shock front is square with the length $R$ and the thickness $\ll R$. The duration of the injection process is $t=R / c$. The injection forms a cube like source. This particular geometry was chosen to describe the light-crossing time effect. The source volume was divided into $10 \times 10 \times 10$ cells. Summing the emissions of the cells in the proper way (detailed description in Chiaberge \& Ghisellini 1999) makes it possible to simulate this effect.

As we already discussed, a single zone model is not able to explain a quadratic or a more than quadratic correlation. Therefore we propose more complex scenario, where the X-ray emission is produced mostly by a relatively large source $(R \sim$ $\left.10^{16} \mathrm{~cm}\right)$. This source has a relatively small particle density and is therefore not able to produce efficient gamma-ray emission. To explain $\mathrm{TeV}$ gamma rays we use another source that is compact $\left(R \sim 10^{15} \mathrm{~cm}\right)$ and dense, and is able to dominate the emission in this energy range. The two sources are completely independent and located at different positions inside the jet. But by chance the emission of both sources is observed at the same time. For simplicity we use the same injection scenario, described above, to calculate the evolution of both sources.

Note that the scenario which assumes the simultaneous emission of two or more sources was already successfully used to explain the rapid TeV variability observed in PKS 2155-304 (Aharonian et al. 2007). Compact sources (of a size of about $10^{14} \mathrm{~cm}$ ) which produce $\mathrm{TeV}$ emission several times stronger than X-ray radiation can explain activity, where the variability time scale is of about a few minutes. The main problem of compact and dense sources - absorption of $\mathrm{TeV}$ emission due to electron-positron par production, is negligible in such an approach (Katarzynski et al. 2008).

The results of our modelling are presented in Fig. 1, where we show spectra selected for two different time moments, the light curves and the obtained correlation. The source size determines directly the variability time scale. Accordingly, a more extended source produces relatively slow variability, whereas the compact source explains the flare. The correlation obtained from the modelling does not perfectly reproduce the observed 

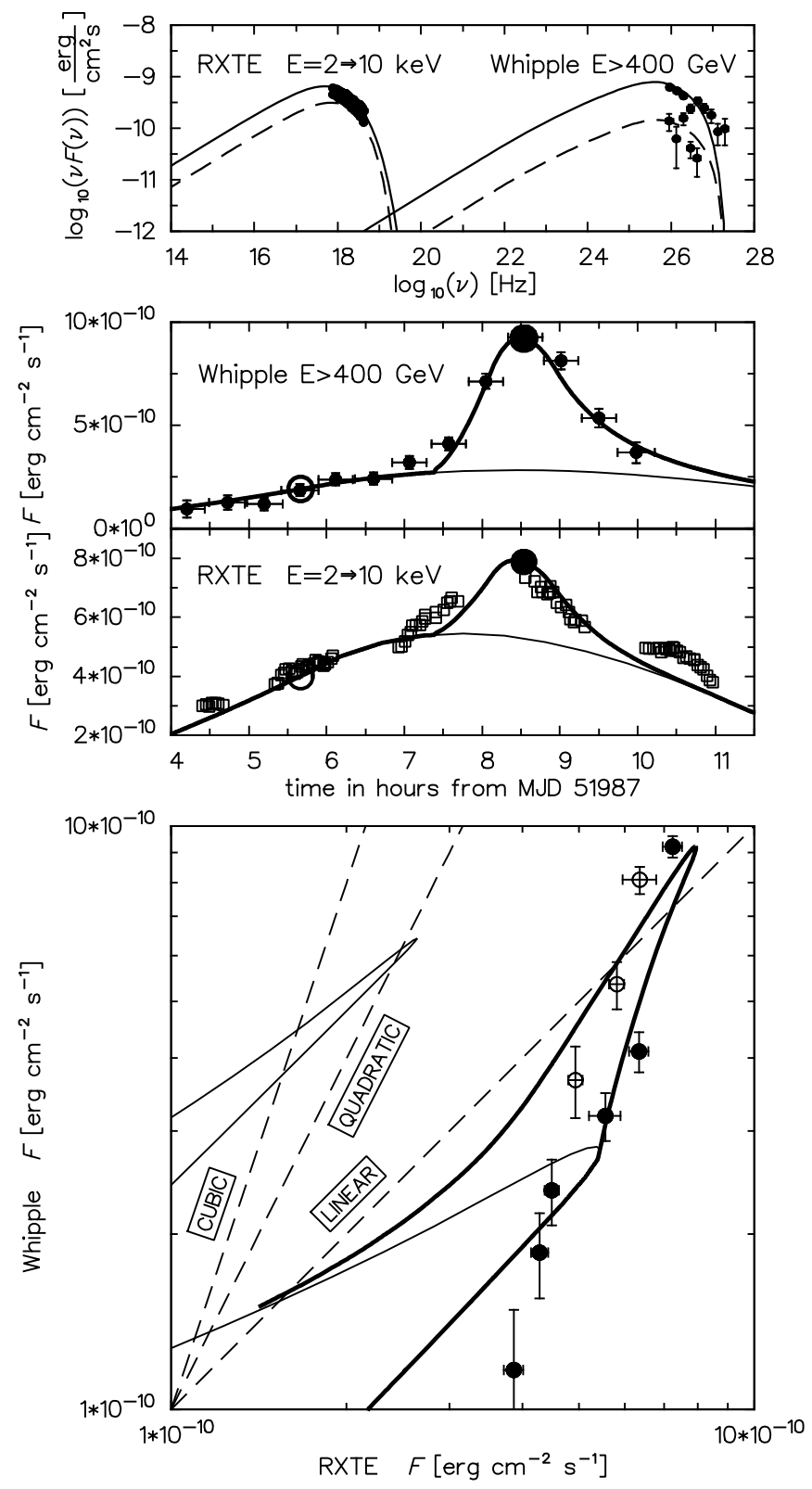

Fig. 1. The activity of Mrk 421 observed by Whipple and RXTE experiments on 19 of March 2001 (Fossati et al. 2008) and the results of our modeling which assumes the particle injection. The upper panel shows spectra obtained for two different time moments: before the main flare and at the top of the flare. The middle panels show the light curves where the total emission is indicated by the thick lines and, the thin lines show only the extended source emission. The dots in the middle panels show the time moments, where the spectra were obtained. Lower panel shows the observed correlation and the results of the modeling, where the open symbols indicate the decay phase and the thin lines show the correlations calculated separately for each source.

relation. The correlation index is different for the rising and decay phase of the activity. This is directly related to the fact that the increment of the emission is produced by the injection, whereas the decay is caused by the radiative cooling. The two completely different physical processes control the evolution during the rise and decay of the activity and the correlation has also different slopes during these phases. In Fig. 1 we show the total correlation obtained for both sources simultaneously and the correlations calculated separately for each source. The correlation calculated for the single source has a linear slope $\left(x_{\uparrow} \simeq 1\right)$ during the rising phase and and an almost square root slope $\left(x_{\downarrow} \simeq 0.5\right)$ in the decay phase. The total correlation shows that the slopes are changing significantly when the emission of two sources is observed simultaneously $\left(x_{\uparrow} \simeq 1 \rightarrow 3\right.$ and $x_{\downarrow} \simeq 0.5 \rightarrow 2$ ). This helps to explain the observed correlation, but the difference between $x_{\uparrow}$ and $x_{\downarrow}$ produced within the single source appears also in the total correlation.

The values of the physical parameters used in the modelling which are identical for both sources are: Doppler factor $\delta=20$, $\gamma_{\min }=1, \gamma_{\max }=10^{6}, n=2$. The difference appears in the source size $R=1.1 \times 10^{16}$ and $1.77 \times 10^{15} \mathrm{~cm}$, the magnetic field strength $B=0.05$ and $0.08 \mathrm{G}$ and the density of the injected particles $t_{\text {inj }} Q_{\text {inj }}=1.7 \times 10^{4}$ and $7 \times 10^{5} \mathrm{~cm}^{-3}$ for the extended and the compact source respectively. The number of free parameters shows that the model we use is quite simple, and this was the main reason to chose this particular scenario. A detailed description of the model is given in Chiaberge \& Ghisellini (1999) and Katarzynski et al. (2008).

Note that we have tested also a scenario which precisely describes the acceleration process (Katarzynski et al. 2006). This particular model gives a different value of the correlation for a single source emission. However, in this case $x_{\uparrow}$ is also significantly different from $x_{\downarrow}$ because two different processes (acceleration and cooling) control the rise and decay of a flare. This appears to be the main problem for the models which are trying to explain the activity by the evolution of the particle energy spectrum. It seems that within all scenarios which assume the particle energy evolution, only adiabatic compression and then adiabatic expansion of the source could give $x_{\uparrow}=x_{\downarrow}$. But, this approach requires a negligible radiative cooling which makes this scenario not realistic.

\section{Doppler boosting effect}

Simultaneous emission of two or more sources can explain any slope of the correlation where the slope depends on the relative value of the X-ray and the gamma-ray emission of the sources. However, the observed correlation seems to be very similar for the rising and decaying phase of a flare. This appears to be problematic for the models that assume different physical processes to explain the two phases of the activity. We decided to test a very simple scenario that assumes changes of the Doppler factor as a main reason of the flux variations. This idea was already proposed several times - for example to explain the activity of Mrk 501 (Villata \& Raiteri 1999).

An intrinsically isotropic emission of a source that travels with the relativistic velocity $V=\beta c$ is confined in a beam along the velocity vector. The half-opening angle of the beam is $\phi \simeq 1 / \Gamma$ in radians, where $\Gamma=1 / \sqrt{1-\beta^{2}}$ is the Lorentz factor. This is the well-know beaming effect that amplifies the observed emission. For a given angle $\theta$ between the velocity and the direction to the observer the amplification of the emission $F_{v}=\delta^{3} F_{v^{\prime}}^{\prime}$ is described by the Doppler factor $\delta=1 /(\Gamma(1-\beta \cos \theta))$. Therefore a small increase or decrease of $\theta$ may cause significant variations of the observed flux. This may happen when the source travels on a helical or quasi-helical trajectory. Such trajectories of the jets were observed many times in AGNs and may cause quasi-periodic activity observed in blazars (e.g. Rani et al. 2009; Qian et al. 2009). Still for the sake of simplicity we do not describe the trajectory of the source in our simulations. This will require many free parameters and is not necessary to test our 
assumptions. We simply describe the change of the viewing angle by the cosine function

$\theta(y)=\theta_{\max }-\Delta \theta \frac{\cos (y)+1}{2}$,

where $\theta_{\max }=180 /(\pi \Gamma)$ and $\Delta \theta$ is the amplitude of the change. Parameter $y$ changes linearly from $-\pi$ to $\pi$ during a flare, where $t_{\mathrm{a}}$ describes the duration of the activity in the observer's frame.

Note that the amplification of the emission is frequencyindependent and will always give a linear correlation $\left(x_{\uparrow}=\right.$ $\left.x_{\downarrow}=1\right)$. This is the main advantage of this approach. Moreover, the correlation slope does not depend on the evolution of the Doppler factor in time. Therefore, the activity can be simulated for example by the source acceleration and deceleration, but this approach requires special justification. We assume a constant value of the source velocity and small changes of the viewing angle that give significant variations of the observed flux. Note that this requires a relatively small value of $\theta<10^{\circ}$ in general.

To describe the observed spectra we use a very simple model of a spherical and homogeneous source with a uniform density and magnetic field. The approach used before which assumes simultaneous emission of a big and small source is also used here to explain the more than linear correlation. We assume the same velocity for both sources that gives $\Gamma=10$, and $\theta_{\max }=5.73 \mathrm{deg}$ and the same amplitude of the viewing angle change $\Delta \theta=4.73$. However, the assumed duration of the activity for the big source is $t_{\mathrm{a}}=18.5 \mathrm{~h}$, whereas for the small one it is $t_{\mathrm{a}}=4.2 \mathrm{~h}$ in the observer's frame. This indicates that the sources are traveling along different trajectories. The difference appears also in the radius $R=10^{16}$ and $1.45 \times 10^{15} \mathrm{~cm}$, the magnetic field strength $B=0.1$ and $0.05 \mathrm{G}$ and the particle density $K=2.5 \times 10^{3}$ and $2 \times 10^{5} \mathrm{~cm}^{-3}$ for the big and small source respectively. The particle energy distribution inside the big source is assumed to be a broken power-law function $N(\gamma)=K \gamma^{-n_{1}}$ for $\gamma<\gamma_{\text {brk }}$ and $N(\gamma)=K \gamma_{\text {brk }}^{n_{2}-n_{1}} \gamma^{-n_{2}}$ above the break. We use $\gamma_{\text {min }}=1, \gamma_{\text {brk }}=$ $2 \times 10^{5}, \gamma_{\max }=10^{6}, n_{1}=2$ and $n_{2}=4$ to describe this spectrum. The energy spectrum inside the small source is approximated by a power-law function, where we use $\gamma_{\min }=1, \gamma_{\max }=2 \times 10^{6}$ and $n=2$. The values used here are very similar to the parameters used in the injection scenario. Note that the intrinsic emission of the sources is constant in time. This means that the sources are in equilibrium, where the radiative cooling is fully compensated by the acceleration. The probability of such a situation is rather low, but this scenario is physically possible. Dominance of the acceleration or the cooling will slightly disturb the perfect linear correlation provided by the Doppler boosting effect. However, to test an ideal case, where for a single source $x_{\uparrow}=x_{\downarrow}$, we assume the equilibrium.

To compare the boosting scenario with the approach presented before we simulate the same activity of Mrk 421. The result of the simulation is presented in Fig. 2. This particular model provides a better fit for the observed spectra and the light curves. However, this is a very simple scenario that has more free parameters. A single source emission provides a perfectly linear correlation which is well visible in the lower part of the correlation panel in Fig. 2, where only the big source emission is correlated. Simultaneous emission of both sources gives almost an cubic correlation. However, the correlation is not the same for the rise and decay of the flare. This is the result of the relative shift in time between the light curves produced by each source. The flare produced by a single source is symmetric in time in this particular scenario. But the maximum of the flare produced by the small source is delayed by about half an hour in comparison to the maximum of the big source activity. This causes
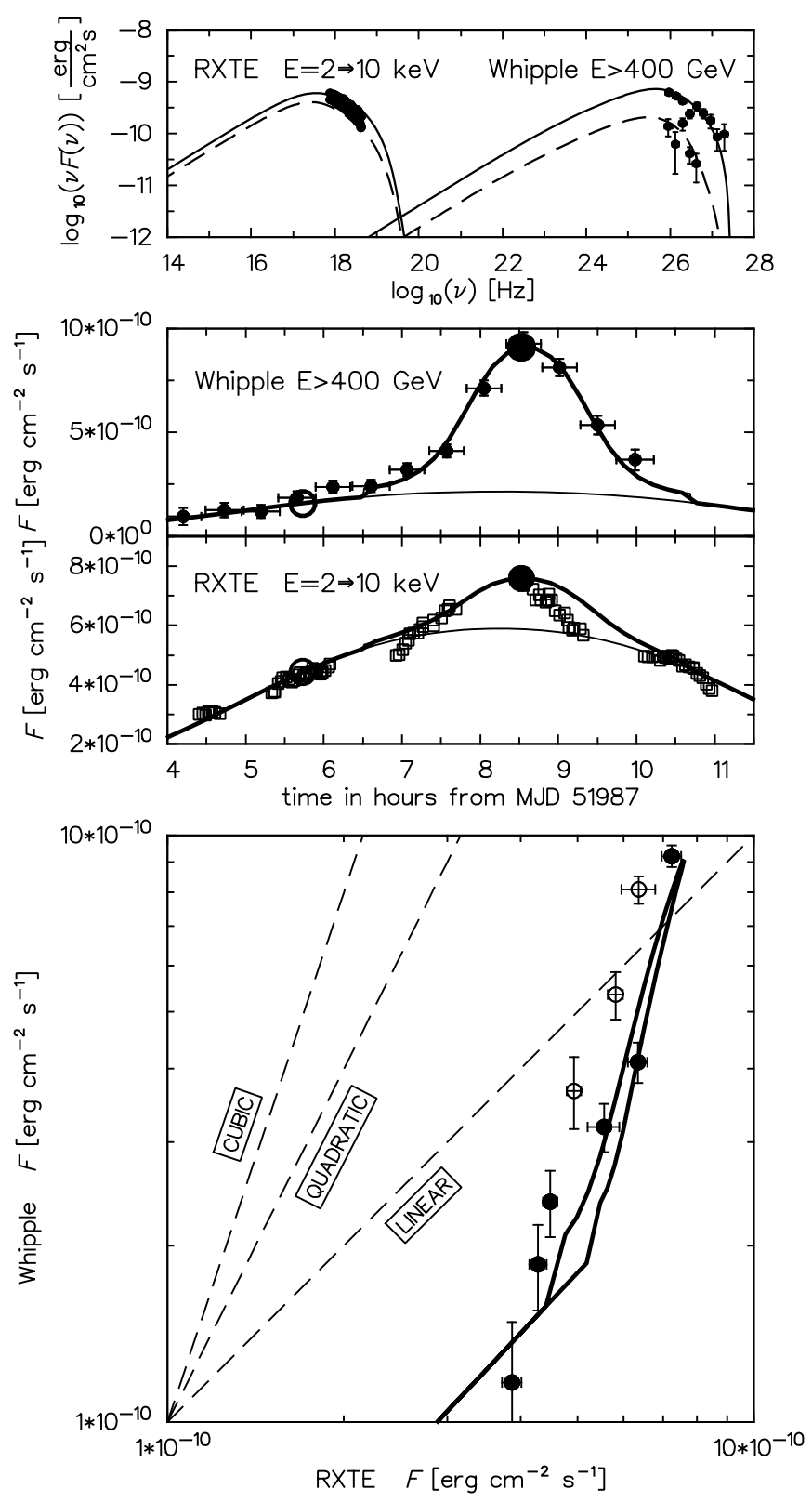

Fig. 2. The same activity of Mrk 421 as in Fig. 1 and the results of our modelling which assume the evolution of the Doppler factor. Note that in this particular case the correlation for a single source is perfectly linear. This is well visible in the bottom part of the correlation panel, where only the background source is contributing to the total emission.

the small difference between $x_{\uparrow}$ and $x_{\downarrow}$. This shows an important fact: that the correlation produced by many independent sources can be diluted simply by the delays between the flares. This may be the reason why the correlation was well determined only in a few cases. Finally, the calculated correlation is slightly shifted in comparison with the observed correlation. This is the result of the delay between the observed X-ray and gamma-ray flare. Such a delay cannot be simulated in our simple scenario.

\section{Summary}

Single zone models frequently used to explain emission of blazars in the $\mathrm{TeV}$ range are not able to explain quadratic or more than quadratic correlation between the X-ray and gammaray emission. So we propose a simple solution to this problem 
which assumes a simultaneous emission of at least two independent sources. In the first approach we use the model that simulates activity by the particle injection. This rather classical approach was successfully applied in many similar models. But our calculations show that the simulated correlation has different slopes for the rising and decay phase of the flare. This seems to be a general problem for the models that assume different processes (e.g. acceleration and cooling) to explain rise and decay of a flare. This lead us to propose an alternative approach, where the activity is produced by the Doppler boosting effect. This scenario provides a perfectly linear correlation for the single source emission in the rising and decaying phase of the activity. But we still have to simulate simultaneous emission of the two sources to obtain quadratic or cubic correlation. The proposed approach has several advantages:

- It can explain any slope of the correlation.

- In the extreme case it is possible to explain the orphan flares.

- The approach does not involve a new model of the emission, it uses the standard SSC scenario to explain a single source radiation.

- It may explain why the correlation was well determined only in a few cases so far.

- In was already shown that using this approach it is possible to explain also the rapid variability.

The correlation may give important information about the region of the jet where the high energy activity is generated. However, many further observations are required to fully understand the nature of the correlation. There are still many open questions. For example, the correlation is observed exceptionally, is this normal for TeV blazars or is this rather a problem with the observation quality? Is there any preferable slope of the correlation? How does the correlation look in different sources? Answers for the above questions and for many others will require precise, simultaneous observations in the X-ray and gamma-ray range.

\section{References}

Aharonian, F., Akhperjanian, A. G., Bazer-Bachi, A. R., et al. 2007, ApJ, 664, 71

Aharonian, F., Akhperjanian, A. G., Anton, G., et al. 2009, A\&A, 502, 749

Albert, J., Aliu, E., Anderhub, H., et al. 2007a, ApJ, 669, 1143

Albert, J., Aliu, E., Anderhub, H., et al. 2007b, ApJ, 663, 125

Blazejowski, M., Blaylock, G., Bond, I. H., et al. 2005, ApJ, 630, 130

Bloom, S. D., \& Marscher, A. P. 1996, ApJ, 461, 657

Bonnoli, G., Ching-Cheng Hsu, Goebel, F., et al. 2009, in 31st international cosmic ray Conference Proceedings, in press [arXiv:0907.0831v1]

Catanese, M., Bradbury, S. M., Breslin, A. C., et al. 1997, ApJ, 487, L143

Chiaberge, M., \& Ghisellini, G. 1999, MNRAS, 306, 551

Coppi, P. S., \& Aharonian, F. A. 1999, ApJ, 521, 33

Dermer, C. D., Sturner, S. J., \& Schlickeiser, R. 1997, ApJS, 109, 103

Donnarumma, I., Vittorini, V., Vercellone, S., et al. 2009, ApJ, 691, 13 Fossati, G., Buckley, J. H., Bond, I. H., et al. 2008, ApJ, 677, 906

Gliozzi, M., Sambruna, R. M., Jung, I., et al. 2006, ApJ, 646, 61

Ghisellini, G., Maraschi, L., \& Dondi, L. 1996, A\&ASS, 120, 503 Gould R. J. 1979, A\&A, 76, 306

Graff, P. B., Georganopoulos, M., Perlman, E. S., et al. 2008, ApJ, 689, 68 G

Horan, D., Acciari, V. A., Bradbury, S. M., et al. 2009, ApJ, 695, 596

Inoue, S., \& Takahara, F. 1996, ApJ, 463, 555

Mastichiadis, A., \& Kirk, J. G. 1997, A\&A, 320, 19

Kataoka, J., Takahashi, T., Makino, F., et al. 2000, ApJ, 528, 243

Katarzynski, K., Sol, H., \& Kus, A. 2001, A\&A, 367, 809

Katarzynski, K., Ghisellini, G., Tavecchio, F., et al. 2005, A\&A, 433, 479

Katarzynski, K., Ghisellini, G., Mastichiadis, A., et al. 2006, A\&A, 453, 47

Katarzynski, K., Lenain, J.-P., Zech, A., et al. 2008, MNRAS, 390, 371

Kirk, J. G., Rieger, F. M., \& Mastichiadis, A. 1998, A\&A, 333, 452

Krawczynski, H., Coppi, P. S., Maccarone, T., \& Aharonian, F. A. 2000, A\&A, 353, 97

Krawczynski, H., Coppi, P. S., \& Aharonian, F. 2002, MNRAS, 336, 721

Krawczynski, H., Hughes, S. B., Horan, D., et al. 2004, ApJ, 601, 151

Pian, E., Vacanti, G., Tagliaferri, G., et al. 1998, ApJ, 492, L17

Qian, Shan-Jie, Witzel, A., Zensus, J. A., et al. 2009, RAA, 9, 137

Sokolov, A., Marscher, A. P., \& McHardy, I. M. 2004, ApJ, 613, 725

Sambruna, R. M., Aharonian, F. A., Krawczynski, H., et al. 2000, ApJ, 538, 127

Takahashi, T., Kataoka, J., Madejski, G., et al. 2000, ApJ, 542, 105

Rani, B., Wiita, P., J., \& Gupta, A. C. 2009, ApJ, 696, 2170

Rees, M. J. 1978, MNRAS, 184, 61

Villata, M., \& Raiteri, C. M. 1999, A\&A, 347, 30 